\title{
Eculizumab treatment for renal failure in a pediatric patient with COVID-19
}

\author{
Ruchi Mahajan $^{1} \cdot$ Marissa Lipton ${ }^{1} \cdot$ Larisa Broglie $^{2} \cdot$ Namrata Gargee Jain $^{1} \cdot$ Natalie Somera Uy ${ }^{1}(\mathbb{C}$
}

Received: 19 June 2020 / Accepted: 3 September 2020 / Published online: 26 September 2020

(C) Italian Society of Nephrology 2020

\begin{abstract}
While there are increasing reports of acute kidney injury among hospitalized adults with COVID-19, there is still limited information on renal complications associated with COVID-19 in children. The cause of kidney involvement in COVID19 is likely multifactorial, and appears to involve a complex process, including complement dysregulation and thrombotic microangiopathy. We present a pediatric case of COVID-19 and renal failure due to thombotic microangiopathy, successfully treated with eculizumab.
\end{abstract}

\section{Introduction}

The number of adults affected by COVID-19 overwhelmingly exceeds pediatric patients, and therefore, the vast majority of studies on renal involvement in COVID-19 have been performed in adults. The incidence and etiology of acute kidney injury (AKI) amongst pediatric patients remains largely unknown. Complement activation has been postulated to underlie some pathophysiology of disease in COVID-19 infections, including thrombotic microangiopathy (TMA). To our knowledge, we present the first pediatric case of COVID-19 and severe AKI due to TMA, treated with eculizumab.

Ruchi Mahajan and Marissa Lipton: co-lead authors, equal contributions.

Natalie Somera Uy

nsu1@cumc.columbia.edu

1 Division of Pediatric Nephrology, Columbia University Vagelos College of Physicians and Surgeons, 622 West 168th Street, PH 17-102B, New York, NY 10032, USA

2 Division of Pediatric Bone Marrow Transplantation, Department of Pediatrics, Columbia University Vagelos College of Physicians and Surgeons, New York, NY 10032, USA

\section{Case presentation}

We present the case of a 14 year old female with obesity and asthma, who was admitted to the Pediatric Intensive Care Unit with fever for 7 days, and complaints of abdominal pain, diarrhea, vomiting, myalgias, and chest pain. On admission, she was febrile, tachypneic, and tachycardic, but normotensive. Initial SARS-CoV-2 PCR was negative. On hospital day 2, she became hypotensive, requiring pressor support, and had worsening respiratory distress with oxygen desaturation, requiring intubation. Repeated SARS COV-2 PCR was indeterminate, and antibody testing (IgG) was positive.

Laboratory results demonstrated elevated inflammatory markers, with ferritin $>100,000 \mathrm{ng} / \mathrm{mL}, \mathrm{CRP}>300 \mathrm{mg} / \mathrm{dL}$, and WBC $33,000 / \mu \mathrm{L}$. Serum creatinine $(\mathrm{Cr})$ on admission was $0.7 \mathrm{mg} / \mathrm{dL}$ (eGFR $85 \mathrm{~mL} / \mathrm{min} / 1.73 \mathrm{~m}^{2}$ by modified Schwartz formula), but she developed oliguric AKI on hospital day 3, and her peak Cr was $8.97 \mathrm{mg} / \mathrm{dL}$ on hospital day 7. Although she was responsive to diuretic treatment with moderate increase in urine output, she developed progressive fluid overload with worsening uremia (BUN $170 \mathrm{mg} / \mathrm{dL}$ ) and metabolic acidosis. Urinalyses demonstrated $100-300 \mathrm{mg} /$ $\mathrm{dL}$ protein with moderate-large blood, with variable RBCs (ranging from 2 to 59/hpf). Creatine kinase (CK) $(55,695$ $\mathrm{U} / \mathrm{L}$, normal 40-308 U/L) and urine myoglobin $(102 \mathrm{mg} / \mathrm{L}$; normal $0-1 \mathrm{mg} / \mathrm{L}$ ) were elevated, suggestive of viral-induced rhabdomyolysis, also contributing to AKI, but CK downtrended as creatinine worsened.

She also had mild thrombocytopenia (platelets 126,000/ $\mathrm{mL}$ ) and hemolytic anemia (nadir $\mathrm{Hgb} 6.8 \mathrm{~g} / \mathrm{dL}$ ), requiring 
multiple pRBC transfusions. Work up of anemia demonstrated normal reticulocyte count and normal haptoglobin, but elevated LDH (4087 U/L; normal 100-275 U/L) and bilirubin $(6.2 \mathrm{mg} / \mathrm{dL}$; normal $0.2-1.3 \mathrm{mg} / \mathrm{dL})$ with schistocytes noted on peripheral smear. ADAMTS13 was mildly low (42, normal 68-163) but not consistent with TTP $(<10)$, and repeat ADAMTS13 was normal (87). Serum complements were low with C3 $33 \mathrm{mg} / \mathrm{dL}$ (normal 80-162 mg/dL) and $\mathrm{C} 44.0 \mathrm{mg} / \mathrm{dL}$ (normal $14-47 \mathrm{mg} / \mathrm{dL}$ ). Other serology tests including ANCA, ANA, dsDNA, ASLO, anti-GBM levels were within normal limits. Renal ultrasound showed normal size and echogenicity of both kidneys, and Doppler study indicated normal vascular flow pattern. Echocardiogram revealed biventricular dysfunction and coronary artery dilatation. Cardiac markers were elevated (proBNP 5600 pg/ $\mathrm{mL}$, troponin $135 \mathrm{ng} / \mathrm{mL}$ ).

She was treated with intravenous pulse steroids for acute myocarditis, and IVIG for coronary ectasia. She received Anakinra for 7 days given concern for severe inflammatory response and cytokine storm. While her inflammatory disease, cardiac markers and hemodynamic status started to improve, she had persistent hemolytic anemia and worsening renal failure. When her BUN/Cr reached 168/7.97 mg/dL on hospital day 10 , continuous renal replacement therapy (CRRT) was initiated. Her pressor requirement dissipated, and she developed elevated blood pressure, treated with hydralazine and a calcium channel blocker. With worsening hypertension, along with persistent renal failure, hemolytic anemia, microscopic hematuria, and proteinuria, TMA was suspected. She also developed bloody endotracheal tube secretions, concerning for pulmonary involvement of TMA. Renal biopsy was not performed due to unstable clinical condition. She was found to have elevated C5b9 level (1669 ng/L; normal<244), and given high suspicion for complement-mediated TMA, she was treated with eculizumab $900 \mathrm{mg}$ IV weekly $\times 3$ doses. CRRT was discontinued after $24 \mathrm{~h}$ of eculizumab initiation, and her renal function returned to baseline after 3 weeks (Fig. 1). Her hemoglobin remained stable, and she did not require further transfusions after initial eculizumab dose. Platelets were slow to recover, and decreased further $(50,000 / \mathrm{mL})$ but improved to $199,000 /$ $\mathrm{mL}$ at discharge. Serum complements normalized. Hypertension, proteinuria and hematuria resolved after 3 weeks. Repeat C5b9 level improved to $275 \mathrm{ng} / \mathrm{L}$ prior to discharge. Eculizumab was discontinued after 3 doses given significant clinical improvement, while genetic testing for aHUS/TMA remains pending.

\section{Discussion}

COVID-19 caused by SARS-CoV-2 virus can cause multiple organ failure including AKI. Reported incidence of AKI in critically ill adult patients in New York has been as high as $36.6 \%$ [1]. The etiology of AKI in COVID-19 patients has not been fully elucidated, and likely goes beyond the common AKI causes often seen in critically ill patients such as nephrotoxic exposures, hemodynamic instability, and decreased renal perfusion. Increasing evidence has suggested additional etiologies, including viral-induced

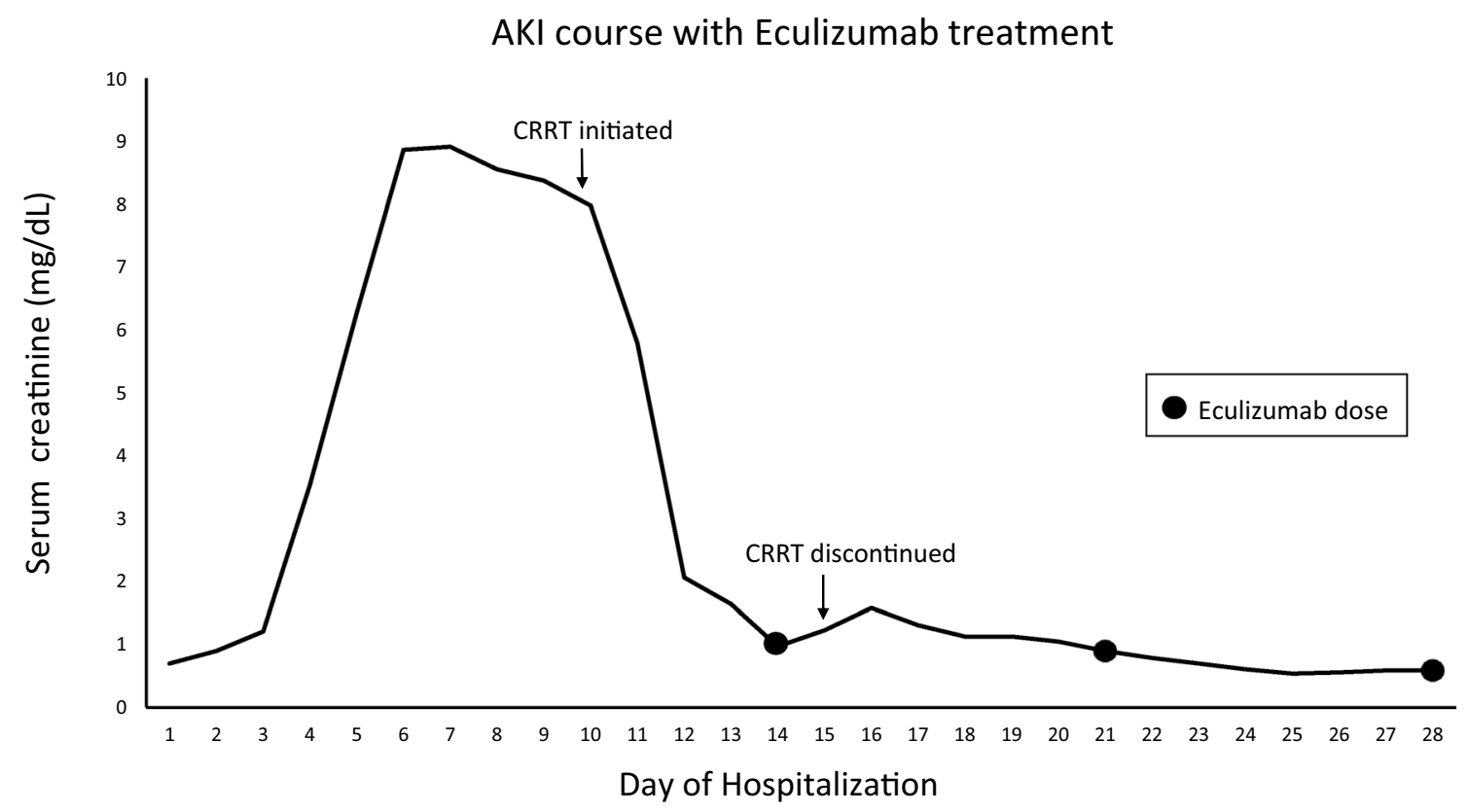

Fig. 1 AKI course and renal recovery with eculizumab treatment. Eculizumab was given weekly on days 14, 21 and 28. CRRT was initiated on day 10 and discontinued on day 15 
rhabdomyolysis, cytokine storm, complement dysregulation, and direct viral invasion [2], which likely contributed to our patient's AKI.

Additionally, critically ill COVID-19 patients have been described to have a high incidence of thrombotic complications [3]. The etiology of these events remains unclear, although studies are ongoing. Histopathology of patients from Wuhan have reported finding microthrombi in pulmonary vasculature on autopsy [4]. A case series from 5 patients in New York demonstrated a pattern of tissue damage consistent with complement-mediated microvascular injury in the lungs and skin [5].

TMA has been implicated in AKI through several etiologies. In pediatric patients, acquired HUS is commonly associated with infection (i.e. Shiga toxin-producing Escherichia coli or Streptococcus pneumoniae) [6]. While TMA is a pathologic diagnosis made by tissue biopsy, it can commonly be inferred clinically, in the observation of microangiopathic hemolytic anemia and thrombocytopenia. In addition, genetic causes of complement-mediated HUS have been described, which lead to complement activation and formation of the membrane attack complex, lysing target cells and resulting in cell death. C3 may be low in complement-mediated HUS, depending on the location of complement pathway disruption and degree of consumption. C5b9 is one of several proteins that make up the membrane attack complex, and a high level is suggestive of activation of the complement pathway. Over activation of the complement pathway also results in damage to endothelium; this, in turn, propagates the coagulation cascade and triggers the formation of microthrombi in vessels and hemolytic anemia. It is the microthrombi that lead to the end organ damage that is seen in HUS.

Eculizumab is a monoclonal antibody against C5, preventing the formation of $\mathrm{C} 5 \mathrm{~b}$ and therefore complement activation. The drug gained FDA approval for treatment of atypical (complement-mediated) HUS (aHUS), and paroxysmal nocturnal hemoglobinuria, with recent expansion to treat neuroinflammatory diseases [7]. Short treatment with eculizumab has also been shown to contribute to improvement in patients with secondary aHUS whose TMA had persisted despite treatment of the underlying cause [8]. In our case, 3 doses of eculizumab were given and discontinued after clinical improvement.

Several studies have suggested that complement activation contributes to COVID-19 disease, and targeting this cascade may be a potential treatment. Complement inhibition in murine studies involving SARS-CoV and MERS$\mathrm{CoV}$ infections have shown promise [9]. Additionally, a case series of critically-ill Italian COVID-19 patients with ARDS produced encouraging results; all four patients who received eculizumab (900 $\mathrm{mg}$, up to 4 doses) have recovered [10]. Eculizumab is currently enrolled in a SOLID-C19 compassionate use study for adult patients in the US [11]. In addition to tissue samples consistent with complementmediated injury in the lungs [5], a study by Diao et al. [12] observed complement $\mathrm{C} 5 \mathrm{~b} 9$ deposition in renal tubules of six patients with SARS-CoV-2 infection, suggestive of cascade presence in the kidney. Thus, we present this case as the first use of eculizumab for complement inhibition in pediatric complement-mediated AKI, associated with COVID-19 infection.

As children account for a very small proportion of patients affected with primary acute COVID-19 infection [13], limited data exist on AKI in this population. Children have been implicated in an emerging condition, Multisystem Inflammatory Syndrome in Children (MIS-C), thought to be a post-viral syndrome associated with COVID-19. MIS-C is a hyperinflammatory state, characterized by persistent fever with a constellation of symptoms including hypotension, elevated inflammatory markers, and multi-organ involvement (e.g., cardiac, gastrointestinal, renal, hematologic, dermatologic and neurologic). Cardiac findings include elevated BNP and troponin levels; and diminished left ventricular systolic function with or without coronary artery changes on echocardiogram [14]. The incidence of $\mathrm{AKI}$ in this syndrome is also unknown, with cases only very recently reported. Our patient displays features of both primary COVID-19 infection and MIS-C [15], and it is not known whether the etiologies of AKI in MIS-C overlap with acute infection, or if the mechanisms differ for patients in a post-viral inflammatory state.

\section{Conclusion}

Complement-mediated TMA should be considered as an etiology for AKI in COVID-19 patients. We present a case of complement cascade inhibition using eculizumab as a strategy for treating severe AKI in a pediatric patient. Further investigations are needed to determine whether it can be generalized that complement inhibition is efficacious in the treatment of renal disease as well as other manifestations of COVID-19.

\section{Compliance with ethical standards}

Conflict of interest On behalf of all authors, the corresponding author states that there is no conflict of interest.

Ethical approval This article does not contain any studies with human participants performed by any of the authors.

Informed consent The parent of the patient has consented to submission of the case report to the journal. 


\section{References}

1. Hirsch JS, Ng JH, Ross DW, Sharma P, Shah HH, Barnett RL, Hazzan AD, Fishbane S, Jhaveri KD, Northwell COVID-19 Research Consortium \& Northwell Nephrology COVID-19 Research Consortium (2020) Acute kidney injury in patients hospitalized with COVID-19. Kidney Int 98(1):209-218. https:// doi.org/10.1016/j.kint.2020.05.006

2. Batlle D, Soler MJ, Sparks MA, Hiremath S, South AM, Welling PA, Swaminathan S, COVID-19 and ACE2 in Cardiovascular, Lung, and Kidney Working Group (2020) Acute kidney injury in COVID-19: emerging evidence of a distinct pathophysiology. J Am Soc Nephrol 31(7):1380-1383. https://doi.org/10.1681/ ASN.2020040419

3. Klok FA, Kruip M, van der Meer NJM, Arbous MS, Gommers D, Kant KM, Kaptein FHJ, van Paassen J, Stals M, Huisman MV, Endeman H (2020) Incidence of thrombotic complications in critically ill ICU patients with COVID-19. Thromb Res 191:145-147. https://doi.org/10.1016/j.thromres.2020.04.041

4. Luo W, Yu H, Gou J, Li X et al (2020) Clinical pathology of critical patient with novel coronavirus pneumonia (COVID-19). Preprints 2020, 2020020407

5. Magro C, Mulvey JJ, Berlin D, Nuovo G, Salvatore S, Harp J, Baxter-Stoltzfus A, Laurence J (2020) Complement associated microvascular injury and thrombosis in the pathogenesis of severe COVID-19 infection: a report of five cases. Transl Res 220:1-13. https://doi.org/10.1016/j.trsl.2020.04.007

6. Szilágyi A, Kiss N, Bereczki C et al (2013) The role of complement in Streptococcus pneumoniae-associated haemolytic uraemic syndrome. Nephrol Dial Transpl 28(9):2237-2245. https:// doi.org/10.1093/ndt/gft198

7. Ricklin D, Mastellos DC, Reis ES, Lambris JD (2018) The renaissance of complement therapeutics. Nat Rev Nephrol 14(1):26-47. https://doi.org/10.1038/nrneph.2017.156

8. Cavero T, Rabasco C, Lopez A et al (2017) Eculizumab in secondary atypical haemolytic uraemic syndrome. Nephrol Dial Transpl 32(3):466-474. https://doi.org/10.1093/ndt/gfw453
9. Campbell CM, Kahwash R (2020) Will complement inhibition be the new target in treating COVID-19-related systemic thrombosis? Circulation 141(22):1739-1741. https://doi.org/10.1161/CIRCU LATIONAHA.120.047419

10. Diurno F, Numis FG, Porta G et al (2020) Eculizumab treatment in patients with COVID-19: preliminary results from real life ASL Napoli 2 Nord experience. Eur Rev Med Pharmacol Sci 24(7):4040-4047. https://doi.org/10.26355/eurrev_202004_20875

11. NIH (2020) Eculizumab (Soliris) in Covid-19 infected patients (SOLID-C19). (Clinicaltrials.gov Identifier NCT04288713). https://clinicaltrials.gov/ct2/show/NCT04288713. Accessed 28 May 2020

12. Diao B, Wang C, Wang R, Feng Z, Tan Y, Wang H, Wang C, Liu L, Liu Y, Wang G, Yuan Z, Ren L, Wu Y, Chen Y (2020) Human kidney is a target for novel severe acute respiratory syndrome coronavirus 2 (SARS-CoV-2) infection. medRxiv 2020.03.04.20031120. https://doi.org/10.1101/2020.03.04.20031 120

13. CDC COVID-19 Response Team (2020) Coronavirus disease 2019 in Children-United States, February 12-April 2, 2020. MMWR Morb Mortal Wkly Rep 69:422-426. https://doi. org/10.15585/mmwr.mm6914e4

14. CDC Health Alert Network (2020) Multisystem inflammatory syndrome in children associated with coronavirus disease 2019. https://emergency.cdc.gov/han/2020/han00432.asp. Accessed 28 May 2020

15. Cheung EW, Zachariah P, Gorelik M, Boneparth A, Kernie SG, Orange JS, Milner JD (2020) Multisystem inflammatory syndrome related to COVID-19 in previously healthy children and adolescents in New York City. JAMA. https://doi.org/10.1001/ jama.2020.10374

Publisher's Note Springer Nature remains neutral with regard to jurisdictional claims in published maps and institutional affiliations. 